\title{
DOUBLE AFFINE HECKE ALGEBRAS FOR THE SPIN SYMMETRIC GROUP
}

\author{
WEIQIANG WANG
}

\begin{abstract}
We introduce a new class (in two versions, $\ddot{\mathfrak{H}}^{\mathfrak{c}}$ and $\ddot{\mathfrak{H}}^{-}$) of rational double affine Hecke algebras (DaHa) associated to the spin symmetric group. We establish the basic properties of the algebras, such as PBW and Dunkl representation, and connections to Nazarov's degenerate affine Hecke-Clifford algebra and to a new degenerate affine Hecke algebra introduced here. We formulate a Morita equivalence between the two versions of rational DaHa's. The trigonometric generalization of the above constructions is also formulated and its relation to the rational counterpart is established.
\end{abstract}

\section{Introduction}

1.1. The double affine Hecke algebras (DaHa) introduced by Cherednik [2] are intimately related to K. Saito's elliptic root systems [12] and the Dunkl operators [4], and they have numerous connections and applications to Macdonald polynomials, integrable systems, and (quantum) affine algebras, etc. The DaHa affords three variants of degeneration: rational, trigonometric, and elliptic. The rational DaHa, which goes back as a special case to Drinfeld [3], has been actively studied in recent years by many authors (see Etingof-Ginzburg [5] and the review of Rouquier [11] for extensive references).

The question addressed here is whether or not a reasonable notion of DaHa's associated to the spin symmetric group of Schur [13] exists. For Coxeter groups, there is a standard procedure to construct the associated Hecke algebras. The spin symmetric group is not a reflection group, and so a priori it is not clear whether such a DaHa should exist and, if it exists, how its characteristic feature looks like.

In this paper we provide a natural construction of rational and trigonometric DaHa's, denoted by $\ddot{\mathfrak{H}}^{\mathfrak{c}}$ and $\ddot{\mathfrak{H}}^{-}$, associated to the spin symmetric group, which not only exhibits new phenomenon but also suggests for further natural generalization. These new algebras afford favorable properties similar to the usual DaHa (see [5] and Suzuki [15]). Instead of constructing directly the DaHa for the spin symmetric group, our idea is to start with a construction of DaHa for a certain double cover of the hyperoctahedral group, which then by some Morita equivalence leads to a construction of the DaHa for the spin symmetric group. The twisted group algebra of this double covering group is a semidirect product $\mathcal{C}_{n} \rtimes \mathbb{C} S_{n}$ between the group algebra of the symmetric group $S_{n}$ and a Clifford algebra $\mathcal{C}_{n}$ in $n$ generators.

Received by the editors October 12, 2007.

2000 Mathematics Subject Classification. Primary 20 C08. 
1.2. We introduce in Section 2 the rational double affine Hecke-Clifford algebra (DaHCa) $\ddot{\mathfrak{H}}^{\mathfrak{c}}$ with parameter $u$ and show that $\ddot{\mathfrak{H}}^{\mathfrak{c}}$ has a standard PBW basis. In particular, $\ddot{\mathfrak{H}}^{\mathfrak{c}}$ admits a triangular decomposition with the middle part being the algebra $\mathcal{C}_{n} \rtimes \mathbb{C} S_{n}$. In contrast to the usual rational DaHa, the symmetry between the two halves of polynomial generators for $\ddot{\mathfrak{H}}^{\mathfrak{c}}$ has to be broken. We further provide a realization of $\ddot{\mathfrak{H}}^{\mathfrak{c}}$ in terms of Dunkl operators.

A degenerate affine Hecke-Clifford algebra $\mathfrak{H}^{\mathfrak{c}}$ was introduced by Nazarov [9] for studying the Young symmetrizer for the spin symmetric group (cf. Brundan-Kleshchev $[1,7]$ for recent development on representations of $\mathfrak{H}^{\mathfrak{c}}$ and see [3] and Lusztig [8] for the usual degenerate/graded affine Hecke algebras). We show that the DaHa $\ddot{\mathfrak{H}}^{\mathfrak{c}}$ contains a family of subalgebras isomorphic to $\mathfrak{H}^{\mathfrak{c}}$, analogous to the usual DaHa setup (cf. $[2,5])$.

We then introduce the rational spin double affine Hecke algebra (sDaHa) $\ddot{\mathfrak{H}}^{-}$associated to the spin symmetric group and parameter $u$. The PBW theorem for $\ddot{\mathfrak{H}}^{-}$ provides the following isomorphism of vector spaces

$$
\ddot{\mathfrak{H}}^{-} \cong \mathcal{C}\left[\xi_{1}, \ldots, \xi_{n}\right] \otimes \mathbb{C} S_{n}^{-} \otimes \mathbb{C}\left[y_{1}, \ldots, y_{n}\right],
$$

where $\mathbb{C} S_{n}^{-}$is the spin symmetric group algebra. A key new feature is that the generators $y_{i}$ 's commute with each other but the $\xi_{i}$ 's anti-commute with each other (cf. Definition 3.1). We also introduce a (new) degenerate spin affine Hecke algebra $\mathfrak{H}^{-}$. The results for $\ddot{\mathfrak{H}}^{-}$and $\mathfrak{H}^{-}$in Section 3 are parallel to those for $\ddot{\mathfrak{H}}^{\mathfrak{c}}$ and $\mathfrak{H}^{\mathfrak{c}}$ in Section 2.

In Section 4 we establish an explicit isomorphism of super algebras

$$
\ddot{\mathfrak{H}}^{\mathfrak{c}} \stackrel{\cong}{\longrightarrow} \mathcal{C}_{n} \otimes \ddot{\mathfrak{H}}^{-} .
$$

In this case, the superalgebras $\ddot{\mathfrak{H}}^{\mathfrak{c}}$ and $\ddot{\mathfrak{H}}^{-}$are said to be Morita super-equivalent (cf. $[7,13.2]$ for a justification of the terminology). We also establish another Morita super-equivalence between $\mathfrak{H}^{\mathfrak{c}}$ and $\mathfrak{H}^{-}$by exhibiting an superalgebra isomorphism $\mathfrak{H}^{\mathfrak{c}} \cong \mathcal{C}_{n} \otimes \mathfrak{H}^{-}$. Such isomorphisms provide a conceptual explanation and easy proofs for the results in Section 3 parallel to Section 2. Without such a connection to $\ddot{\mathfrak{H}}^{\mathfrak{c}}$, even the definition of $\ddot{\mathfrak{H}}^{-}$is by no means obvious. A finite-dimensional version of such isomorphisms, discovered independently in Sergeev [14] and Yamaguchi [16], explained for the well-known fact that the representation theory of the spin symmetric group is (essentially) equivalent to that of the algebra $\mathcal{C}_{n} \rtimes S_{n}$. The three isomorphisms in the finite, affine, and double affine setups are compatible with each other.

We finally introduce the trigonometric DaHCa $\ddot{\mathfrak{H}}_{t r}^{c}$ and the trigonometric sDaHa $\ddot{\mathfrak{H}}_{t r}^{-}$. We construct explicitly a superalgebra isomorphism

$$
\ddot{\mathfrak{H}}_{t r}^{c} \cong \mathfrak{C}_{n} \otimes \ddot{\mathfrak{H}}_{t r}^{-} .
$$

We further establish the precise connections between our rational and trigonometric DaHa's, following Suzuki [15] in the usual DaHa setup. This is developed in Section 5.

1.3. This paper is a first step of a program in which we attempt to develop a new theory of spin Hecke algebras. The new features of the constructions in this paper will be instrumental in telling us how to relax the usual setup (cf. $[3,5]$ and Ram-Shepler [10] for the degenerate case) in order to define appropriate spin (degenerate/double) affine Hecke algebras more generally. The algebras $\ddot{\mathfrak{H}}^{\mathfrak{c}}$ and $\ddot{\mathfrak{H}}^{-}$have large centers, and 
are expected to have rich finite-dimensional representation theory (compare [5] and Gordon [6]).

\section{The rational double affine Hecke-Clifford algebras}

2.1. The symmetric group $S_{n}$. Recall that the symmetric group $S_{n}$ is generated by $s_{i}(1 \leq i \leq n-1)$ subject to the relations:

$$
\begin{aligned}
s_{i} s_{j} & =s_{j} s_{i}, \quad|i-j|>1 \\
s_{i}^{2}=1, \quad s_{i} s_{i+1} s_{i} & =s_{i+1} s_{i} s_{i+1} .
\end{aligned}
$$

Let $s_{i j}$ denote the transposition of $i$ and $j$ in the symmetric group $S_{n}$.

\subsection{The rational DaHCa.}

Definition 2.1. Let $u \in \mathbb{C}$. The rational double affine Hecke-Clifford algebra (or simply, DaHCa) $\ddot{\mathfrak{H}}^{\mathfrak{c}}$ is the $\mathbb{C}$-algebra generated by $x_{i}, y_{i}, c_{i}(1 \leq i \leq n)$ and $S_{n}$, subject to the following relations:

$$
\begin{gathered}
x_{i} x_{j}=x_{j} x_{i}, \quad y_{i} y_{j}=y_{j} y_{i}, \quad(\forall i, j) \\
\sigma x_{i}=x_{\sigma i} \sigma, \quad \sigma y_{i}=y_{\sigma i} \sigma \quad\left(\sigma \in S_{n}\right) \\
c_{i} x_{i}=-x_{i} c_{i}, \quad c_{i} y_{i}=y_{i} c_{i}, \\
c_{j} x_{i}=x_{i} c_{j}, \quad c_{j} y_{i}=y_{i} c_{j}, \quad(i \neq j) \\
\sigma c_{i}=c_{\sigma i} \sigma\left(\sigma \in S_{n}\right), \quad c_{i}^{2}=1, \quad c_{i} c_{j}=-c_{j} c_{i}(i \neq j) \\
{\left[y_{j}, x_{i}\right]=u\left(1+c_{j} c_{i}\right) s_{i j}, \quad(i \neq j)} \\
{\left[y_{i}, x_{i}\right]=-u \sum_{k \neq i}\left(1+c_{k} c_{i}\right) s_{k i} .}
\end{gathered}
$$

Denote by $\mathcal{C}_{n}$, or $\mathcal{C}\left(c_{1}, \ldots, c_{n}\right)$, the Clifford algebra generated by $c_{1}, \ldots, c_{n}$. The rational DaHCa $\ddot{\mathfrak{H}}^{\mathfrak{c}}$ is a super (i.e. $\mathbb{Z}_{2}$-graded) algebra with $\left|c_{i}\right|=\overline{1}$ and $\left|x_{i}\right|=\left|y_{i}\right|=$ $\left|s_{i j}\right|=\overline{0}$. Unlike the usual rational DaHa, the symmetry between $x$ 's and $y$ 's is broken in the definition of $\ddot{\mathfrak{H}}^{\mathrm{c}}$, cf. (2.2).

For $u=0$, we have $\ddot{\mathfrak{H}}_{\mid u=0}^{\mathrm{c}}=\left(\mathbb{C}[\underline{x}, \underline{y}] \tilde{\otimes} \mathfrak{C}_{n}\right) \# S_{n}$, where the tilde refers to the unusual

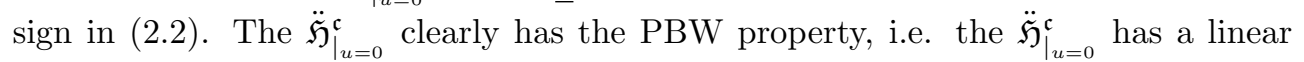
basis $\left\{\underline{x} \underline{\underline{a}} \underline{y^{\underline{b}}} \sigma \underline{\underline{\epsilon}}\right\}$, where $\sigma \in S_{n}, \underline{a}, \underline{b} \in \mathbb{Z}_{+}^{n}, \underline{\epsilon} \in\{0,1\}^{n}$ and $\underline{x}^{\underline{a}}$ denotes the monomials $x_{1}^{a_{1}} \cdots x_{n}^{a_{n}}$ etc. The algebras $\ddot{\mathfrak{H}}^{\mathfrak{c}}$ are isomorphic for all $u \neq 0$.

Theorem 2.2. The PBW property holds for $\ddot{\mathfrak{H}}^{\mathfrak{c}}$. That is, the multiplication of the subalgebra gives rise to an isomorphism of vector spaces:

$$
\mathbb{C}\left[x_{1}, \ldots, x_{n}\right] \otimes \mathbb{C} S_{n} \otimes \mathcal{C}_{n} \otimes \mathbb{C}\left[y_{1}, \ldots, y_{n}\right] \stackrel{\cong}{\rightrightarrows} \ddot{\mathfrak{H}}^{\mathfrak{c}} .
$$

Proof. This can be proved in a similar way as in [5, Proof of Th. 1.3, pp.256-7] (and see loc. cit. for earlier works on PBW algebras), with one crucial modification. Set $V=\mathbb{C}^{2 n}$ with the standard coordinates $\left\{x_{i}, y_{i}\right\}$, and note that $K=\mathcal{C}_{n} \rtimes \mathbb{C} S_{n}$ is a semisimple algebra. The new key observation is that $E:=V \otimes_{\mathbb{C}} K$ is naturally a $K$-bimodule even though $K$ does not act on $V$. The right action of $K$ is via the right multiplication $g: v \otimes a \mapsto v \otimes(a g)$. The $S_{n}$ acts on the left by $\sigma: v \otimes a \mapsto v^{\sigma} \otimes(\sigma a)$, 
while $\mathcal{C}_{n}$ acts by $c_{i}: x_{j} \otimes a \mapsto(-1)^{\delta_{i j}} x_{j} \otimes\left(c_{i} a\right)$ and $c_{i}: y_{j} \otimes a \mapsto y_{j} \otimes\left(c_{i} a\right)$. The rest of the proof is the same as in loc. cit.

Remark 2.3. The observation in the above proof, when applied to more general pairs $(V, K)$, leads to a generalization of Drinfeld's setup [3]. The proof above also shows that $\ddot{\mathfrak{H}}^{\mathfrak{c}}$ is the only 'quadratic' deformation of $\ddot{\mathfrak{H}}_{\mid u=0}^{c}$ with PBW property. Note the special feature of $\ddot{\mathfrak{H}}^{\mathfrak{c}}$ that an additional term $t \cdot 1$ which appeared in the symplectic reflection algebra [5] is not allowed in (2.5) by the conjugation invariance with respect to $c_{i}$.

Remark 2.4. Introducing an additional central element $z$ such that $z^{2}=1$ to $\ddot{\mathfrak{H}}^{\mathfrak{c}}$, we can define a modified algebra $\ddot{\mathfrak{H}}^{\text {mod }}$ with relations

$$
c_{i} c_{j}=z c_{j} c_{i}, \quad c_{i} x_{i}=z x_{i} c_{i}
$$

replacing the corresponding relations in $(2.3)$ and $(2.2)$. Then $\ddot{\mathfrak{H}}^{\text {mod }} /\langle z+1\rangle \cong \ddot{\mathfrak{H}}^{\mathrm{c}}$. On the other hand, $\ddot{\mathfrak{H}}^{\text {mod }} /\langle z-1\rangle$ is isomorphic to the ordinary rational DaHa (cf. [5]) associated to the reflection group $\mathbb{Z}_{2}^{n} \rtimes S_{n}$ with specialized parameters. A similar remark applies to the algebra $\ddot{\mathfrak{H}}^{-}$introduced in Section 3.

Below we always assume that $u \neq 0$ unless otherwise specified.

2.3. Degenerate affine Hecke-Clifford algebra. The degenerate affine HeckeClifford algebra was introduced by Nazarov (also called affine Sergeev algebra in [9]). It is the algebra $\mathfrak{H}^{\mathfrak{c}}$ generated by $a_{i}, c_{i}(i=1, \ldots, n)$ and $S_{n}$, subject to the relations (2.1), (2.3) and the following relations:

$$
\begin{aligned}
a_{j} s_{i} & =s_{i} a_{j} \quad(j \neq i, i+1), \\
a_{i+1} s_{i}-s_{i} a_{i} & =1-c_{i+1} c_{i}, \\
c_{i} a_{i}= & -a_{i} c_{i}, \\
a_{i} a_{j}=a_{j} a_{i}, & c_{j} a_{i}=a_{i} c_{j}, \quad(i \neq j) .
\end{aligned}
$$

Our convention $c_{i}^{2}=1$ is consistent with [7], not with [9]. The $\mathfrak{H}^{\mathfrak{c}}$ is a superalgebra with $c_{i}$ being odd and $a_{i}$ being even for each $i$. For $1 \leq i \leq n$, set

$$
M_{i}:=\sum_{k<i}\left(1-c_{i} c_{k}\right) s_{k i},
$$

The $M_{i}$ 's are the Jucys-Murphy elements introduced in [9] and they satisfy

$$
M_{i} M_{j}=M_{j} M_{i}, \quad(\forall i, j) .
$$

Proposition 2.5. [9]

(1) The algebra $\mathfrak{H}^{\mathfrak{c}}$ admits the PBW property. That is, the multiplication of the subalgebras induces a vector space isomorphism

$$
\mathbb{C}\left[a_{1}, \ldots, a_{n}\right] \otimes \mathbb{C} S_{n} \otimes \mathcal{C}_{n} \stackrel{\cong}{\longrightarrow} \mathfrak{H}^{\mathfrak{c}} .
$$

(2) There exists a unique algebra homomorphism $\mathfrak{H}^{\mathfrak{c}} \rightarrow \mathcal{C}_{n} \rtimes \mathbb{C} S_{n}$, which restricts to the identity map on the subalgebra $\mathfrak{C}_{n} \rtimes \mathbb{C} S_{n}$ of $\mathfrak{H}^{\mathfrak{c}}$, and sends $a_{1}$ to 0 . Moreover, this homomorphism sends each $a_{i}$ to $M_{i}(1 \leq i \leq n)$.

(3) The even center of the algebra $\mathfrak{H}^{\mathfrak{c}}$ is $\mathbb{C}\left[a_{1}^{2}, \ldots, a_{n}^{2}\right]^{S_{n}}$. 
The intertwining elements

$$
\phi_{i}:=s_{i}\left(a_{i}^{2}-a_{i+1}^{2}\right)+\left(a_{i}+a_{i+1}\right)+c_{i} c_{i+1}\left(a_{i}-a_{i+1}\right), \quad 1 \leq i \leq n-1
$$

were introduced in [9] (cf. [7, Chapter 14]).

Proposition 2.6. [9] The intertwining elements $\phi_{i}$ 's satisfy

$$
\begin{aligned}
\phi_{i}^{2} & =2 a_{i}^{2}+2 a_{i+1}^{2}-\left(a_{i}^{2}-a_{i+1}^{2}\right)^{2} \\
\phi_{i} \phi_{i+1} \phi_{i} & =\phi_{i+1} \phi_{i} \phi_{i+1} \\
\phi_{i} \phi_{j} & =\phi_{j} \phi_{i} \quad(|i-j|>1) .
\end{aligned}
$$

\subsection{The affine Hecke-Clifford subalgebra of $\ddot{\mathfrak{H}}^{\mathfrak{c}}$. Set}

$$
z_{i}:=u^{-1} y_{i} x_{i}+M_{i}
$$

Lemma 2.7. For all $i, j$ and each $\alpha \in \mathbb{C}$, the following identities hold:

$$
\begin{aligned}
{\left[z_{i}, z_{j}\right] } & =0, \\
{\left[x_{i}, z_{j}\right]-\left[x_{j}, z_{i}\right] } & =0, \\
{\left[\alpha x_{i}+z_{i}, \alpha x_{j}+z_{j}\right] } & =0 .
\end{aligned}
$$

Proof. We may assume $i<j$. Then, by (2.7), we have

$$
\begin{aligned}
u\left[z_{i}, z_{j}\right]= & u^{-1}\left[y_{i} x_{i}, y_{j} x_{j}\right]+\left[y_{i} x_{i}, M_{j}\right] \\
= & u^{-1}\left(y_{i}\left[x_{i}, y_{j}\right] x_{j}+y_{j}\left[y_{i}, x_{j}\right] x_{i}\right)+\left[y_{i} x_{i}, s_{i j}-c_{j} c_{i} s_{i j}\right] \\
= & -y_{i}\left(s_{i j}-c_{i} c_{j} s_{i j}\right) x_{j}+y_{j}\left(s_{i j}+c_{i} c_{j} s_{i j}\right) x_{i} \\
& +\left(y_{i} x_{i}-y_{j} x_{j}\right) s_{i j}-\left(y_{i} x_{i}+y_{j} x_{j}\right) c_{j} c_{i} s_{i j}=0 .
\end{aligned}
$$

To prove (2.9) for $i<j$, we calculate that

$$
\begin{aligned}
& {\left[x_{i}, z_{j}\right]-\left[x_{j}, z_{i}\right] } \\
= & {\left[x_{i}, u^{-1} y_{j} x_{j}+M_{j}\right]-\left[x_{j}, u^{-1} y_{i} x_{i}\right] } \\
= & u^{-1}\left[x_{i}, y_{j}\right] x_{j}+\left[x_{i}, s_{i j}-c_{j} c_{i} s_{i j}\right]-u^{-1}\left[x_{j}, y_{i}\right] x_{i} \\
= & \left(c_{i} c_{j} s_{i j}-s_{i j}\right) x_{j}+\left(x_{i}-x_{j}\right) s_{i j}-\left(x_{i}+x_{j}\right) c_{j} c_{i} s_{i j}+\left(s_{i j}+c_{i} c_{j} s_{i j}\right) x_{i}=0 .
\end{aligned}
$$

Now (2.10) follows from (2.8) and (2.9).

We remark that $\left[y_{i}, z_{j}\right]-\left[y_{j}, z_{i}\right] \neq 0 \quad(i \neq j)$.

Lemma 2.8. The following identities hold:

$$
c_{i} z_{i}=-z_{i} c_{i}, \quad c_{i} z_{j}=z_{j} c_{i} \quad(i \neq j) .
$$

Proof. Recall $z_{i}=u^{-1} y_{i} x_{i}+M_{i}$. It is known [9] that the Jucys-Murphy elements satisfy $c_{i} M_{i}=-M_{i} c_{i}$, and $c_{j} M_{i}=M_{i} c_{j}$ for $i \neq j$. Clearly, $c_{i}\left(y_{i} x_{i}\right)=-\left(y_{i} x_{i}\right) c_{i}$, and $c_{j}\left(y_{i} x_{i}\right)=\left(y_{i} x_{i}\right) c_{j}$ for $i \neq j$. Now the lemma follows.

Lemma 2.9. The following identities hold:

$$
\begin{aligned}
z_{i+1} s_{i}-s_{i} z_{i} & =1-c_{i+1} c_{i} \\
\left(\alpha x_{i+1}+z_{i+1}\right) s_{i}-s_{i}\left(\alpha x_{i}+z_{i}\right) & =1-c_{i+1} c_{i} .
\end{aligned}
$$


Proof. The second identity follows from the first one and the equation $x_{i+1} s_{i}=s_{i} x_{i}$. Note that

$$
\begin{aligned}
z_{i+1} s_{i}-s_{i} z_{i} & =\left(u^{-1} y_{i+1} x_{i+1}+M_{i+1}\right) s_{i}-s_{i}\left(u^{-1} y_{i} x_{i}+M_{i}\right) \\
& =M_{i+1} s_{i}-s_{i} M_{i}=1-c_{i+1} c_{i}
\end{aligned}
$$

This proves the first identity.

The next theorem follows now from Lemmas 2.7, 2.8, and 2.9.

Theorem 2.10. Fix $\alpha \in \mathbb{C}$. The subalgebra of $\ddot{\mathfrak{H}}^{\mathfrak{c}}$ generated by $c_{i}, \alpha x_{i}+z_{i}(1 \leq i \leq n)$ and $S_{n}$ is isomorphic to the degenerate affine Hecke-Clifford algebra $\mathfrak{H}^{\mathfrak{c}}$.

2.5. The Dunkl operator for $\ddot{\mathfrak{H}}^{\mathfrak{c}}$. The PBW Theorem 2.2 provides the triangular decomposition of $\ddot{\mathfrak{H}}^{\mathfrak{c}}$ :

$$
\ddot{\mathfrak{H}}^{\mathfrak{c}} \cong \mathbb{C}[\underline{x}] \otimes\left(\mathcal{C}_{n} \rtimes \mathbb{C} S_{n}\right) \otimes \mathbb{C}[\underline{y}]
$$

where $\mathbb{C}[\underline{x}]$ is the short-hand for $\mathbb{C}\left[x_{1}, \ldots, x_{n}\right]$, etc. Denote by $\mathfrak{H}_{x}^{\mathfrak{c}}$ the subalgebra of $\ddot{\mathfrak{H}}^{\mathfrak{c}}$ generated by $\mathcal{C}_{n} \rtimes \mathbb{C} S_{n}$ and $x_{1}, \ldots, x_{n}$. The subalgebra $\mathfrak{H}_{y}^{\mathfrak{c}}$ is similarly defined. Take any $\mathcal{C}_{n} \rtimes \mathbb{C} S_{n}$-module $W$, and extend it to an $\mathfrak{H}_{x}^{\mathfrak{c}}$-module with the trivial action of $x_{i}$ 's. Consider the induced $\ddot{\mathfrak{H}}^{\mathfrak{c}}$-module $\operatorname{Ind}_{\mathfrak{H}_{x}^{\mathfrak{c}}}^{\ddot{\mathfrak{H}}^{\mathfrak{c}}} W$, which as a vector space is isomorphic to $\mathbb{C}\left[y_{1}, \ldots, y_{n}\right] \otimes W$. Denote the action by $\circ$. The most interesting $S_{n} \ltimes \mathcal{C}_{n}$-module is the so-called basic spin module $L_{n}=\mathcal{C}\left(c_{1}, \ldots, c_{n}\right)$ of $S_{n} \ltimes \mathcal{C}_{n}$, (where $\mathcal{C}_{n}$ acts by left multiplication and $S_{n}$ acts by permuting the $c_{i}$ 's), and the induced $\ddot{\mathfrak{H}}^{\mathfrak{c}}$-module $\operatorname{Ind}_{\tilde{H}_{x}^{\mathfrak{c}}}^{\ddot{\mathfrak{c}}^{\mathrm{c}}} L_{n}$ is then identified as $\mathbb{C}\left[y_{1}, \ldots, y_{n}\right] \otimes \mathcal{C}\left(c_{1}, \ldots, c_{n}\right)$. Given operators $h$ and $g$, $\frac{h}{g}$ stands for $g^{-1} h$ throughout the paper.

Theorem 2.11. Given a $\mathcal{C}_{n} \rtimes \mathbb{C} S_{n}$-module $W$, the action of $x_{i}$ on $\mathbb{C}\left[y_{1}, \ldots, y_{n}\right] \otimes W$ is realized as "Dunkl operator" as follows. For any polynomial $f=f(\underline{y})$ and $w \in W$, we have

$$
x_{i} \circ(f \otimes w)=u \sum_{k \neq i} \frac{\left(1-s_{k i}\right)(f)}{y_{i}-y_{k}} \otimes\left(1-c_{i} c_{k}\right) s_{k i}(w) .
$$

Proof. We calculate that

$$
x_{i} \circ(f \otimes w)=\left[x_{i}, f\right] \circ w+f x_{i} \circ w=\left[x_{i}, f\right] \circ w .
$$

Now the theorem follows from Lemma 2.12 (2) below.

Lemma 2.12. (1) For $a \in \mathbb{N}$ and $i \neq j$, we have

$$
\begin{aligned}
& {\left[x_{i}, y_{j}^{a}\right]=-u \frac{y_{i}^{a}-y_{j}^{a}}{y_{i}-y_{j}}\left(1-c_{i} c_{j}\right) s_{i j}} \\
& {\left[x_{i}, y_{i}^{a}\right]=u \sum_{k \neq i} \frac{y_{i}^{a}-y_{k}^{a}}{y_{i}-y_{k}}\left(1-c_{i} c_{k}\right) s_{k i} .}
\end{aligned}
$$

(2) Let $f(\underline{y})$ be any polynomial in $y_{1}, \ldots, y_{n}$. Then,

$$
\left[x_{i}, f(\underline{y})\right]=u \sum_{k \neq i} \frac{\left(1-s_{k i}\right) f(\underline{y})}{y_{i}-y_{k}}\left(1-c_{i} c_{k}\right) s_{k i} .
$$


Proof. (1) is proved by induction on $a$ using $\left[x_{i}, y_{j}^{a}\right]=\left[x_{i}, y_{j}^{a-1}\right] y_{j}+y_{j}^{a-1}\left[x_{i}, y_{j}\right]$.

(2) It suffices to check for every monomial $f$. We first prove the case when $f=$ $\prod_{j \neq i} y_{j}^{a_{j}}$ using the first identity in (1). Then we combine with the second identity in (1) to prove for a general monomial $f$ involving powers of $y_{i}$.

Note that the symmetry between $x$ 's and $y$ 's in our algebra $\ddot{\mathfrak{H}}^{\mathfrak{c}}$ is broken. We compute below the Dunkl operator for $y_{i}$.

Lemma 2.13. For $a \in \mathbb{N}$ and $1 \leq i \neq j \leq n$, we have

$$
\begin{aligned}
& {\left[y_{i}, x_{i}^{a}\right]=u \sum_{k \neq i}\left(-\frac{x_{k}^{a}-x_{i}^{a}}{x_{k}-x_{i}} s_{k i}+\frac{x_{i}^{a}-\left(-x_{k}\right)^{a}}{x_{k}+x_{i}} c_{i} s_{k i} c_{i}\right)} \\
& {\left[y_{i}, x_{j}^{a}\right]=u \frac{x_{j}^{a}-x_{i}^{a}}{x_{j}-x_{i}} s_{i j}+u \frac{x_{j}^{a}-\left(-x_{i}\right)^{a}}{x_{j}+x_{i}} c_{i} s_{i j} c_{i} .}
\end{aligned}
$$

Proof. Follows by induction on $a$.

We can identify $\mathbb{C}\left[x_{1}, \cdots, x_{n}\right] \otimes W$ with the induced $\ddot{\mathfrak{H}}^{\mathrm{c}}$-module $\operatorname{Ind}_{\mathfrak{H}_{y}^{\mathrm{c}}}^{\ddot{\mathfrak{H}}^{\mathrm{c}}} W$, where $W$ is a $\mathbb{C} S_{n} \ltimes \mathcal{C}_{n}$-module and is then extended to an $\mathfrak{H}_{y}^{\mathfrak{c}}$-module trivially.

Proposition 2.14. The action of $y_{i}$ on $\mathbb{C}\left[x_{1}, \cdots, x_{n}\right] \otimes W$ is given as follows. For any polynomial $f(\underline{x})$ and $w \in W$, we have

$$
y_{i} \circ(f \otimes w)=\sum_{k \neq i}\left(\frac{f-s_{k i}(f)}{x_{k}-x_{i}} \otimes s_{k i}(w)+\frac{f c_{i} c_{k}-c_{i} c_{k} s_{k i}(f)}{x_{k}+x_{i}} \otimes s_{i k}(w)\right) .
$$

Proof. First note that $y_{i}$ commutes with all $c_{j}$ 's. So we are reduced to the case $f=1$ and $w$ being a monomial. This follows by induction from Lemma 2.13.

2.6. The center of $\ddot{\mathfrak{H}}^{\mathfrak{c}}$. Denote by $Z(A)$ the even center of a superalgebra $A$, which consists of all the even central elements in $A$.

Proposition 2.15. (1) For $u=0$, the even center $\mathcal{Z}\left(\ddot{\mathfrak{H}}_{\left.\right|_{u=0} ^{c}}^{c}\right)$ of the superalgebra $\ddot{\mathfrak{H}}_{\left.\right|_{u=0}}^{\mathfrak{c}}$ is $\mathbb{C}\left[x_{1}^{2}, \ldots, x_{n}^{2} ; y_{1}, \ldots, y_{n}\right]^{\Delta S_{n}}$, where $\Delta$ denotes the diagonal action on $x_{j}^{2}$ 's and $y_{j}$ 's.

(2) We have $\mathbb{C}\left[x_{1}^{2}, \ldots, x_{n}^{2}\right]^{S_{n}} \subset \mathcal{Z}\left(\ddot{\mathfrak{H}}^{\mathfrak{c}}\right)$ and $\mathbb{C}\left[y_{1}, \ldots, y_{n}\right]^{S_{n}} \subset \mathcal{Z}\left(\ddot{\mathfrak{H}}^{\mathfrak{c}}\right), \forall u \in \mathbb{C}$.

Proof. (1) is clear.

(2) By Lemma $2.12(1)$, for each $i$ we have that

$$
\left[x_{i}, y_{1}^{k}+\cdots+y_{n}^{k}\right]=0, \quad \forall k \geq 1 .
$$

By definition of $\ddot{\mathfrak{H}}^{\mathfrak{c}}, y_{1}^{k}+\cdots+y_{n}^{k}$ commutes with $\mathbb{C} S_{n} \ltimes \mathcal{C}_{n}$. So $y_{1}^{k}+\cdots+y_{n}^{k} \in \mathcal{Z}\left(\ddot{\mathfrak{H}}^{\mathfrak{c}}\right)$ for all $k$, and thus $\mathbb{C}\left[y_{1}, \ldots, y_{n}\right]^{S_{n}} \subset z\left(\ddot{\mathfrak{H}}^{\mathfrak{c}}\right)$. We have $\mathbb{C}\left[x_{1}^{2}, \ldots, x_{n}^{2}\right]^{S_{n}} \subset \mathcal{Z}\left(\ddot{\mathfrak{H}}^{\mathfrak{c}}\right)$, by arguing similarly using Lemma 2.13 and the definition of $\ddot{\mathfrak{H}}^{\mathfrak{c}}$.

Example 2.16. For $n=2, x_{1}^{2} y_{1}+x_{2}^{2} y_{2}-\left(x_{1}+x_{2}\right) s_{12}-c_{1}\left(x_{1}+x_{2}\right) s_{12} c_{1} \in \mathcal{Z}\left(\ddot{\mathfrak{H}}^{\mathfrak{c}}\right)$. 


\section{The rational spin double affine Hecke algebras}

The results in this section are presented in a way parallel to Section 2, but with all proofs omitted except for the PBW theorem. They can be either proved in the same way as for their counterparts in Section 2, or follow directly from the counterparts in Section 2 via the isomorphisms to be established in Section 4.

3.1. The spin symmetric group algebra. The symmetric group $S_{n}$ affords a double cover $\widetilde{S}_{n}$, nontrivial for $n \geq 4$, according to Schur [13] (cf. [7]):

$$
1 \longrightarrow \mathbb{Z}_{2} \longrightarrow \widetilde{S}_{n} \longrightarrow S_{n} \longrightarrow 1 \text {. }
$$

Denote $\mathbb{Z}_{2}=\{1, z\}$. The spin symmetric group algebra $\mathbb{C} S_{n}^{-}:=\mathbb{C}\left[\widetilde{S}_{n}\right] /\langle z+1\rangle$ is generated by $t_{i}, i=1, \cdots, n-1$ subject to the following relations:

$$
\begin{aligned}
t_{i}^{2}=1, & t_{i} t_{i+1} t_{i}=t_{i+1} t_{i} t_{i+1}, \\
t_{i} t_{j}=-t_{j} t_{i}, \quad & |i-j|>1 .
\end{aligned}
$$

The algebra $\mathbb{C} S_{n}^{-}$is naturally a superalgebra by declaring $t_{i}$ for every $i$ to be odd.

Define the "transpositions" of odd parity, for $1 \leq i<j \leq n$,

$$
[i, j]=-[j, i]=(-1)^{j-i-1} t_{j-1} \cdots t_{i+1} t_{i} t_{i+1} \cdots t_{j-1}
$$

which satisfies the following relations:

$$
\begin{aligned}
{[i, i+1]=t_{i}, } & {[i, j]^{2}=1, } \\
t_{i}[i, j] t_{i}=-[i+1, j], & \text { for } j \neq i, i+1 .
\end{aligned}
$$

Define the odd Jucys-Murphy elements

$$
\mathrm{M}_{j}=\sum_{k<j}[k, j], \quad(1 \leq j \leq n) .
$$

We have [14] (also see [7, Chapter 13])

$$
\left[\mathrm{M}_{i}, \mathrm{M}_{j}\right]_{+}:=\mathrm{M}_{i} \mathrm{M}_{j}+\mathrm{M}_{j} \mathrm{M}_{i}=0, \quad(i \neq j) .
$$

As usual, we have denoted that $[a, b]_{+}=a b+b a$.

\subsection{The rational sDaHa.}

Definition 3.1. Let $u \in \mathbb{C}$. The rational spin double affine Hecke algebra (sDaHa) is the algebra $\ddot{\mathfrak{H}}^{-}$generated by $\xi_{i}, y_{i}(1 \leq i \leq n)$ and $t_{i}(1 \leq i \leq n-1)$, subject to the relations (3.1) for $t_{i}$ and the following relations:

$$
\begin{aligned}
\xi_{i} \xi_{j}=-\xi_{j} \xi_{i}, & y_{i} y_{j}=y_{j} y_{i}, \quad(i \neq j), \\
t_{i} \xi_{i}=-\xi_{i+1} t_{i}, & t_{i} y_{i}=y_{i+1} t_{i}, \\
t_{j} \xi_{i}=-\xi_{i} t_{j}, & t_{j} y_{i}=y_{i} t_{j}, \quad(i \neq j, j+1), \\
{\left[y_{i}, \xi_{j}\right]=} & u[i, j], \quad(i \neq j), \\
{\left[y_{i}, \xi_{i}\right]=} & u \sum_{k \neq i}[i, k] .
\end{aligned}
$$

Denote by $\mathcal{C}\left[\xi_{1}, \ldots, \xi_{n}\right]$ the algebra generated by $\xi_{1}, \ldots, \xi_{n}$ subject to the relations $\xi_{i} \xi_{j}=-\xi_{j} \xi_{i}$ for $i \neq j$. Clearly, it has a linear basis $\underline{\xi}^{\text {a }}$, where $\underline{\mathbf{a}} \in \mathbb{Z}_{+}^{n}$. 
Theorem 3.2. The PBW property holds for $\ddot{\mathfrak{H}}^{-}$. That is, the multiplication of the subalgebras gives rise to an isomorphism of vector spaces:

$$
\mathcal{C}\left[\xi_{1}, \ldots, \xi_{n}\right] \otimes \mathbb{C} S_{n}^{-} \otimes \mathbb{C}\left[y_{1}, \ldots, y_{n}\right] \stackrel{\cong}{\longrightarrow} \ddot{\mathfrak{H}}^{-} .
$$

Proof. It follows readily from the definition that $\ddot{\mathfrak{H}}^{-}$is spanned by the elements $\underline{\xi}^{\underline{a}} \sigma \underline{y}^{\underline{b}}$, where $\sigma$ runs over a basis for $\mathbb{C} S_{n}^{-}$and $\underline{a}, \underline{b} \in \mathbb{Z}_{+}^{n}$. Recall the homomorphism $\Psi$ in Theorem 4.2. The images $\Psi\left(\underline{\xi}^{\underline{a}} \sigma \underline{y}^{\underline{b}}\right)$ in explicit formulas are clearly all linearly independent in $\ddot{\mathfrak{H}}^{\mathfrak{c}}$ by the PBW Theorem 2.2 for $\ddot{\mathfrak{H}}^{\mathfrak{c}}$. Thus, all the elements $\underline{\xi}^{\underline{a}} \sigma \underline{y}^{\underline{b}}$ are linearly independent in $\ddot{\mathfrak{H}}^{-}$.

Remark 3.3. Note the subtle signs in the definition of $\ddot{\mathfrak{H}}^{-}$. The algebra $\ddot{\mathfrak{H}}^{-}$is naturally a superalgebra with $y_{i}$ being even and $\xi_{i}, t_{i}$ being odd for each $i$. The algebras $\ddot{\mathfrak{H}}^{-}$ are all isomorphic for different $u \neq 0$.

Below we assume that $u \neq 0$ unless otherwise specified.

3.3. The degenerate spin affine Hecke algebra. The degenerate spin affine Hecke algebra is the algebra $\mathfrak{H}^{-}$generated by $b_{i}(1 \leq i \leq n)$ and $t_{i}(1 \leq i \leq n-1)$, subject to the relations (3.1) for $t_{i}$ and the following relations:

$$
\begin{aligned}
b_{i} b_{j} & =-b_{j} b_{i}, \quad(i \neq j) \\
b_{i+1} t_{i} & =-t_{i} b_{i}+1, \\
t_{j} b_{i} & =-b_{i} t_{j}, \quad(i \neq j, j+1) .
\end{aligned}
$$

The algebra $\mathfrak{H}^{-}$has a superalgebra structure with both $b_{i}$ and $t_{i}$ being odd elements for every $i$. Note that the algebra $\mathfrak{H}^{-}$contains $\mathcal{C}\left[b_{1}, \ldots, b_{n}\right]$ and $\mathbb{C} S_{n}^{-}$as subalgebras.

Proposition 3.4. (1) The algebra $\mathfrak{H}^{-}$admits the PBW property. That is, the multiplication of the subalgebras induces a vector space isomorphism

$$
\mathcal{C}\left[b_{1}, \ldots, b_{n}\right] \otimes \mathbb{C} S_{n}^{-} \stackrel{\cong}{\longrightarrow} \mathfrak{H}^{-} .
$$

(2) There exists a unique algebra homomorphism $\mathfrak{H}^{-} \rightarrow \mathbb{C} S_{n}^{-}$, which extends the identity map on the subalgebra $\mathbb{C} S_{n}^{-}$of $\mathfrak{H}^{-}$and sends $b_{1}$ to 0 . Moreover, this homomorphism sends each $b_{i}$ to $M_{i}(1 \leq i \leq n)$.

(3) The even center of the superalgebra $\mathfrak{H}^{-}$is $\mathbb{C}\left[b_{1}^{2}, \ldots, b_{n}^{2}\right]^{S_{n}}$.

We define the (odd) intertwining elements in $\mathfrak{H}^{-}$:

$$
\psi_{i}:=t_{i}\left(b_{i}^{2}-b_{i+1}^{2}\right)-\left(b_{i}-b_{i+1}\right), \quad 1 \leq i \leq n-1 .
$$

Proposition 3.5. The intertwining elements $\psi_{i}$ 's satisfy that

$$
\begin{aligned}
\psi_{i}^{2} & =b_{i}^{2}+b_{i+1}^{2}-\left(b_{i}^{2}-b_{i+1}^{2}\right)^{2} \\
\psi_{i} \psi_{i+1} \psi_{i} & =\psi_{i+1} \psi_{i} \psi_{i+1} \\
\psi_{i} \psi_{j}= & -\psi_{j} \psi_{i}(|i-j|>1) \\
\psi_{i} b_{j}= & -b_{j} \psi_{i}(j \neq i, i+1) \\
\psi_{i} b_{i}=-b_{i+1} \psi_{i}, & \psi_{i} b_{i+1}=-b_{i} \psi_{i} .
\end{aligned}
$$




\subsection{The spin affine Hecke subalgebra of $\ddot{\mathfrak{H}}^{-}$. Let}

$$
\mathfrak{z}_{i}:=u^{-1} y_{i} \xi_{i}+\mathrm{M}_{i} .
$$

Lemma 3.6. For $i \neq j$, the following identities hold:

$$
\begin{aligned}
{\left[\mathfrak{z}_{i}, \mathfrak{z}_{j}\right]_{+} } & =0, \\
{\left[\xi_{i}, \mathfrak{z}_{j}\right]_{+}+\left[\xi_{j}, \mathfrak{z}_{i}\right]_{+} } & =0, \\
{\left[\alpha \xi_{i}+\mathfrak{z}_{i}, \alpha \xi_{j}+\mathfrak{z}_{j}\right]_{+} } & =0, \quad \forall \alpha \in \mathbb{C} .
\end{aligned}
$$

Lemma 3.7. The following identities hold:

$$
\left(\alpha \xi_{i+1}+\mathfrak{z}_{i+1}\right) t_{i}=-t_{i}\left(\alpha \xi_{i}+\mathfrak{z}_{i}\right)+1, \quad \forall \alpha \in \mathbb{C} .
$$

Theorem 3.8. Fix $\alpha \in \mathbb{C}$. The subalgebra of $\ddot{\mathfrak{H}}^{-}$generated by $\alpha \xi_{i}+\mathfrak{z}_{i}(1 \leq i \leq n)$ and $\mathbb{C} S_{n}^{-}$is isomorphic to the degenerate spin affine Hecke algebra $\mathfrak{H}^{-}$.

3.5. The Dunkl operators for $\ddot{\mathfrak{H}}^{-}$. Denote by $\mathfrak{H}_{\xi}^{-}$the subalgebra of $\ddot{\mathfrak{H}}^{-}$generated by $\mathbb{C} S_{n}^{-}$and $\xi_{1}, \ldots, \xi_{n}$. Take any $\mathbb{C} S_{n}^{-}$-module $W$, and extend it to an $\mathfrak{H}_{\xi}^{-}$module with the trivial action of $\xi_{i}$ 's. Consider the induced $\ddot{\mathfrak{H}}^{-}$-module $\operatorname{Ind}_{\mathfrak{H}_{\xi}^{-}}^{\ddot{\mathfrak{H}}^{-}} W \cong$ $\mathbb{C}\left[y_{1}, \ldots, y_{n}\right] \otimes W$.

Theorem 3.9. The action of $\xi_{i} \in \ddot{\mathfrak{H}}^{-}$on $\mathbb{C}\left[y_{1}, \ldots, y_{n}\right] \otimes W$ is realized as "Dunkl operator" as follows. For any polynomial $f=f(y)$ and $w \in W$, we have

$$
\xi_{i} \circ(f \otimes w)=u \sum_{k \neq i} \frac{\left(1-s_{k i}\right)(f)}{y_{i}-y_{k}} \otimes[k, i](w) .
$$

\subsection{The center of $\ddot{\mathfrak{H}}^{-}$.}

Proposition 3.10. (1) For $u=0$, the even center $\mathcal{Z}\left(\ddot{\mathfrak{H}}_{\left.\right|_{u=0} ^{-}}\right)$of the superalgebra $\ddot{\mathfrak{H}}_{\mid}^{-}$is $\mathbb{C}\left[\xi_{1}^{2}, \ldots, \xi_{n}^{2} ; y_{1}, \ldots, y_{n}\right]^{\Delta S_{n}}$, where $\Delta$ denotes the diagonal action on the $\xi_{j}^{2}$ 's and $y_{j}$ 's.

(2) For any $u \in \mathbb{C}, \mathbb{C}\left[\xi_{1}^{2}, \ldots, \xi_{n}^{2}\right]^{S_{n}} \subset \mathcal{Z}\left(\ddot{\mathfrak{H}}^{-}\right)$and $\mathbb{C}\left[y_{1}, \ldots, y_{n}\right]^{S_{n}} \subset \mathcal{Z}\left(\ddot{\mathfrak{H}}^{-}\right)$.

Example 3.11. For $n=2, \xi_{1}^{2} y_{1}+\xi_{2}^{2} y_{2}+2\left(\xi_{1}-\xi_{2}\right) t_{1} \in \mathcal{Z}\left(\ddot{\mathfrak{H}}^{-}\right)$.

\section{The algebra isomorphisms}

The results in this section use earlier notations but their proofs will be independent of Section 3. Note that the multiplication in a tensor product $\mathcal{Q} \otimes \ddot{\mathfrak{H}}^{-}$of two super algebras $\mathcal{C}$ and $\ddot{\mathfrak{H}}^{-}$has a suitable sign convention:

$$
\left(c^{\prime} \otimes b^{\prime}\right)(c \otimes b)=(-1)^{\left|b^{\prime}\right||c|}\left(c^{\prime} c \otimes b^{\prime} b\right) .
$$

We shall write a typical element in $\mathcal{C} \otimes \ddot{\mathfrak{H}}^{-}$as $c b$ rather than $c \otimes b$, and use short-hand notations $c=c \otimes 1, b=1 \otimes b$.

Theorem 4.1. There exists a superalgebra isomorphism:

$$
\begin{gathered}
\widehat{\Phi}: \mathfrak{H}^{\mathfrak{c}} \stackrel{\cong}{\longrightarrow} \mathcal{C}_{n} \bigotimes \mathfrak{H}^{-}, \\
c_{i} \mapsto c_{i}, a_{i} \mapsto \sqrt{-2} c_{i} b_{i}, s_{i} \mapsto \frac{1}{\sqrt{-2}}\left(c_{i}-c_{i+1}\right) t_{i} .
\end{gathered}
$$


The inverse map is given by

$$
\widehat{\Psi}: c_{i} \mapsto c_{i}, b_{i} \mapsto \frac{1}{\sqrt{-2}} c_{i} a_{i}, t_{i} \mapsto \frac{1}{\sqrt{-2}}\left(c_{i+1}-c_{i}\right) s_{i} .
$$

Proof. Clearly $\widehat{\Phi}, \widehat{\Psi}$ are inverses to each other, once we show that they are algebra homomorphisms. Note that $\mathcal{C}_{n} \rtimes \mathbb{C} S_{n}$ is a subalgebra of $\mathfrak{H}^{\mathfrak{c}}$ and $\widehat{\Phi}$ extends the isomorphism established in $[14,16]$

$$
\Phi^{f i n}: \mathcal{C}_{n} \rtimes \mathbb{C} S_{n} \longrightarrow \mathcal{C}_{n} \otimes \mathbb{C} S_{n}^{-} .
$$

This takes care of all the defining relations for the images of $\widehat{\Phi}$ or $\widehat{\Psi}$ of the generators which do not involve $a_{i}$ or $b_{i}$ (the verification of these relations can be done directly without difficulty). Among the defining relations involving $b_{i}$, the most complicated one is $\widehat{\Psi}\left(b_{i+1} t_{i}+t_{i} b_{i}\right)=1$, which is equivalent to the following identity in $\mathfrak{H}^{\text {c }}$ :

$$
\frac{1}{\sqrt{-2}} c_{i+1} a_{i+1} \cdot \frac{1}{\sqrt{-2}}\left(c_{i+1}-c_{i}\right) s_{i}+\frac{1}{\sqrt{-2}}\left(c_{i+1}-c_{i}\right) s_{i} \cdot \frac{1}{\sqrt{-2}} c_{i} a_{i}=1 .
$$

This can be checked directly using the defining relations $(2.6)$ of $\mathfrak{H}^{\mathfrak{c}}$. The verification of the remaining relations is similar, and will be skipped.

An argument similar to the above implies the following isomorphism theorem.

Theorem 4.2. There exists a superalgebra isomorphism:

$$
\begin{gathered}
\Phi: \ddot{\mathfrak{H}}^{\mathfrak{c}} \stackrel{\cong}{\cong} \mathcal{C}_{n} \bigotimes \ddot{\mathfrak{H}}^{-}, \\
y_{i} \mapsto y_{i}, c_{i} \mapsto c_{i}, x_{i} \mapsto \sqrt{-2} c_{i} \xi_{i}, s_{i} \mapsto \frac{1}{\sqrt{-2}}\left(c_{i}-c_{i+1}\right) t_{i} .
\end{gathered}
$$

The inverse map is given by

$$
\Psi: y_{i} \mapsto y_{i}, c_{i} \mapsto c_{i}, \xi_{i} \mapsto \frac{1}{\sqrt{-2}} c_{i} x_{i}, t_{i} \mapsto \frac{1}{\sqrt{-2}}\left(c_{i+1}-c_{i}\right) s_{i} .
$$

Remark 4.3. (1) Recall the intertwining elements $\phi_{i}$ and $\psi_{i}$ from Sections 2 and 3. The isomorphism $\widehat{\Phi}$ sends $\phi_{i}$ to $-\sqrt{-2}\left(c_{i}-c_{i+1}\right) \psi_{i}$ for every $i$.

(2) Recall from Propositions 2.10 and 3.8 that $\alpha x_{i}+z_{i}$ (resp. $\alpha \xi_{i}+\mathfrak{z}_{i}$ ) are the 'polynomial' generators of a degenerate affine Hecke algebra isomorphic to $\mathfrak{H}^{\mathfrak{c}}$ (resp. $\left.\mathfrak{H}^{-}\right)$. The $\Phi$ sends $\alpha x_{i}+z_{i}$ to $\sqrt{-2} c_{i}\left(\alpha \xi_{i}+\mathfrak{z}_{i}\right)$ for each $i$. This is compatible with the fact that $\widehat{\Phi}$ sends $a_{i}$ to $\sqrt{-2} c_{i} b_{i}$.

(3) Note also that $\widehat{\Phi}$ or $\Phi$ sends the JM element $M_{i}$ to $\sqrt{-2} c_{i} \mathrm{M}_{i}$, which amounts to checking the identity $\Phi\left(\frac{1}{\sqrt{-2}}\left(c_{i}-c_{k}\right) s_{i k}\right)=[k, i]$.

\section{The trigonometric version of $\mathrm{DaHCa}$ and $\mathrm{sDaHa}$}

5.1. The trigonometric DaHCa. We start with the following definition. 
Definition 5.1. The trigonometric double affine Hecke-Clifford algebra is the algebra $\ddot{\mathfrak{H}}_{t r}^{\mathfrak{c}}$ generated by $e^{ \pm \epsilon_{i}}, \epsilon_{i}^{\vee}, c_{i}(1 \leq i \leq n)$ and $S_{n}$, subject to the following relations:

$$
\begin{aligned}
& c_{i}^{2}=1, c_{i} c_{j}=-c_{j} c_{i} \quad(i \neq j) \\
& e^{\epsilon_{i}} e^{-\epsilon_{i}}=1, e^{\epsilon_{i}} e^{\epsilon_{j}}=e^{\epsilon_{j}} e^{\epsilon_{i}}, \\
& \epsilon_{i}^{\vee} \epsilon_{j}^{\vee}=\epsilon_{j}^{\vee} \epsilon_{i}^{\vee}, c_{j} e^{\epsilon_{i}}=e^{\epsilon_{i}} c_{j} \quad(\forall i, j), \\
& \sigma e^{\epsilon_{i}}=e^{\epsilon_{\sigma i}} \sigma, \sigma c_{i}=c_{\sigma i} \sigma \quad\left(\sigma \in S_{n}\right), \\
& c_{i} \epsilon_{i}^{\vee}=-\epsilon_{i}^{\vee} c_{i}, c_{j} \epsilon_{i}^{\vee}=\epsilon_{i}^{\vee} c_{j} \quad(i \neq j), \\
& \epsilon_{i+1}^{\vee} s_{i}-s_{i} \epsilon_{i}^{\vee}=u\left(1-c_{i+1} c_{i}\right), \epsilon_{j}^{\vee} s_{i}=s_{i} \epsilon_{j}^{\vee} \quad(j \neq i, i+1), \\
& {\left[\epsilon_{i}^{\vee}, e^{\eta}\right]=u \sum_{k \neq i} \operatorname{sgn}(k-i) \frac{e^{\eta}-e^{s_{k i}(\eta)}}{1-e^{\operatorname{sgn}(k-i) \cdot\left(\epsilon_{k}-\epsilon_{i}\right)}}\left(1-c_{i} c_{k}\right) s_{k i} . }
\end{aligned}
$$

The algebra $\ddot{\mathfrak{H}}_{t r}^{\mathrm{c}}$ admits a natural superalgebra structure with $c_{i}$ being odd and all other generators being even. Note that the subalgebra generated by $e^{\epsilon_{i}}(1 \leq i \leq n)$, denoted by $\mathbb{C}[P]$, is identified with the group algebra of the weight lattice of type $G L_{n}$; the subalgebra generated by $e^{\epsilon_{i}}(1 \leq i \leq n)$ and $S_{n}$ is identified with the group algebra of the extended affine Weyl group of type $G L_{n}$; for $u \neq 0$, the subalgebra generated by $u^{-1} \epsilon_{i}^{\vee}, c_{i}(1 \leq i \leq n)$ and $S_{n}$ is identified with the degenerate affine Hecke-Clifford algebra (cf. Sect. 2.3).

Theorem 5.2. (1) There exists a unique superalgebras homomorphism $\iota: \ddot{\mathfrak{H}}^{\mathfrak{c}} \rightarrow$ $\ddot{\mathfrak{H}}_{t r}^{\mathrm{c}}$, which extends the identity map on the subalgebra $\mathfrak{C}_{n} \rtimes \mathbb{C} S_{n}$ such that

$$
\begin{aligned}
& \iota\left(y_{i}\right)=e^{\epsilon_{i}} \\
& \iota\left(x_{i}\right)=e^{-\epsilon_{i}}\left(\epsilon_{i}^{\vee}-u \sum_{k<i}\left(1-c_{i} c_{k}\right) s_{k i}\right) .
\end{aligned}
$$

Moreover, $\iota$ is injective.

(2) The homomorphism ८ extends to an algebra isomorphism

$$
\mathbb{C}\left[\underline{y}^{ \pm}\right] \otimes_{\mathbb{C}[\underline{y}} \ddot{\mathfrak{H}}^{\mathfrak{c}} \stackrel{\cong}{\longrightarrow} \ddot{\mathfrak{H}}_{t r}^{\mathfrak{c}} .
$$

The inverse $j$ is an extension of the identity map on $\mathcal{C}_{n} \rtimes \mathbb{C} S_{n}$ such that

$$
\begin{aligned}
j\left(e^{\epsilon_{i}}\right) & =y_{i}, \\
j\left(\epsilon_{i}^{\vee}\right) & =y_{i} x_{i}+u \sum_{k<i}\left(1-c_{i} c_{k}\right) s_{k i} .
\end{aligned}
$$

Proof. The $j$ and (the extended) $\iota$ are clearly inverses to each other, once we show that they are algebra homomorphisms.

Recall from (2.4) that $u z_{i}=y_{i} x_{i}+u \sum_{k<i}\left(1-c_{i} c_{k}\right) s_{k i}$. Let us verify

$$
j\left(\left[\epsilon_{i}^{\vee}, e^{\epsilon_{i}}\right]\right)=\left[j\left(\epsilon_{i}^{\vee}\right), j\left(e^{\epsilon_{i}}\right)\right]=\left[u z_{i}, y_{i}\right] .
$$

By a long but straightforward computation we have that

$$
\begin{aligned}
& {\left[u z_{i}, y_{j}\right]=-u y_{i}\left(1-c_{i} c_{j}\right) s_{i j}, \quad(i<j),} \\
& {\left[u z_{i}, y_{j}\right]=-u y_{j}\left(1-c_{i} c_{j}\right) s_{i j}, \quad(i>j),} \\
& {\left[u z_{i}, y_{i}\right]=u \sum_{k>i} y_{i}\left(1-c_{i} c_{k}\right) s_{k i}+u \sum_{k<i}\left(1-c_{i} c_{k}\right) s_{k i} y_{i} .}
\end{aligned}
$$


By another direct computation, we see that the commutator $\left[\epsilon_{i}^{\vee}, e^{\epsilon_{j}}\right]$ are given by exactly the right-hand side of $\left[u z_{i}, y_{j}\right]$ above with $y_{i}$ therein replaced by $e^{\epsilon_{i}}$, for $i<j$, $i=j$, or $i>j$.

The verification of other relations is easier and will be skipped.

Remark 5.3. Theorem 5.2 and Theorem 5.6 below are analogous to the relation between the usual rational DaHa and trigonometric DaHa due to Suzuki [15].

Proposition 5.4. The PBW property holds for $\ddot{\mathfrak{H}}_{t r}^{c}$. That is, the multiplication of the subalgebras gives rise to an isomorphism of vector spaces:

$$
\mathbb{C}[P] \otimes \mathbb{C} S_{n} \otimes \mathcal{C}_{n} \otimes \mathbb{C}\left[\epsilon_{1}^{\vee}, \ldots, \epsilon_{n}^{\vee}\right] \stackrel{\cong}{\longrightarrow} \ddot{\mathfrak{H}}_{t r}^{c} .
$$

Proof. Clearly the multiplication homomorphism is surjective.

The injectivity of this homomorphism follows from Theorem 5.2 (2) and the PBW property for $\ddot{\mathfrak{H}}^{\mathfrak{c}}$ (and thus for $\mathbb{C}\left[\underline{y}^{ \pm}\right] \otimes_{\mathbb{C}[y]} \ddot{\mathfrak{H}}^{\mathfrak{c}}$ ). More explicitly, $j$ sends $\epsilon_{i}{ }^{\vee}$ to $y_{i} x_{i}$ plus lower terms. Via $j$ and with such lower terms ignored, any linear relation among $\underline{\epsilon}^{\vee} \underline{a} \sigma \underline{\underline{e}} \underline{\underline{e}} y^{\underline{b}}$ is translated to the same relation among $(y x) \underline{\underline{a}} \sigma \underline{\underline{e}}^{\underline{e}} \underline{y}^{\underline{b}}$. But we know the latter are all linearly independent.

\subsection{The trigonometric sDaHa.}

Definition 5.5. The trigonometric spin double affine Hecke algebra is the algebra $\ddot{\mathfrak{H}}_{t r}^{-}$generated by $e^{ \pm \epsilon_{i}}, \zeta_{i}(1 \leq i \leq n)$ and $t_{i}(1 \leq i \leq n-1)$, subject to the relations (3.1) for $t_{i}$ and the following relations:

$$
\begin{aligned}
e^{\epsilon_{i}} e^{-\epsilon_{i}}=1, & e^{\epsilon_{i}} e^{\epsilon_{j}}=e^{\epsilon_{j}} e^{\epsilon_{i}}, \\
t_{i} e^{\epsilon_{i}}=e^{\epsilon_{i+1}} t_{i}, & t_{i} e^{\epsilon_{j}}=e^{\epsilon_{j}} t_{i} \quad(j \neq i, i+1), \\
\zeta_{i+1} t_{i}+t_{i} \zeta_{i}=u, & \zeta_{j} t_{i}=-t_{i} \zeta_{j}(j \neq i, i+1), \\
\zeta_{i} \zeta_{j}= & -\zeta_{j} \zeta_{i}(i \neq j), \\
{\left[\zeta_{i}, e^{\eta}\right]=} & u \sum_{k \neq i} \operatorname{sgn}(k-i) \frac{e^{\eta}-e^{s_{k i}(\eta)}}{1-e^{\operatorname{sgn}(k-i) \cdot\left(\epsilon_{k}-\epsilon_{i}\right)}}[k, i] .
\end{aligned}
$$

Note that the algebra $\ddot{\mathfrak{H}}_{t r}^{-}$is naturally a superalgebra by declaring $e^{ \pm \epsilon_{i}}$ to be even and $\zeta_{i}$ and $t_{i}$ to be odd for every $i$. For $u \neq 0$, the subalgebra generated by $\zeta_{i}$ $(1 \leq i \leq n)$ and $\mathbb{C} S_{n}^{-}$is identified with the degenerate spin affine Hecke algebra $\mathfrak{H}^{-}$ (cf. Sect. 3.3).

Theorem 5.6. (1) There exists a unique homomorphism of superalgebras $\iota^{-}$: $\ddot{\mathfrak{H}}^{-} \rightarrow \ddot{\mathfrak{H}}_{\text {tr }}^{-}$, which extends the identity map on the subalgebra $\mathbb{C} S_{n}^{-}$such that

$$
\begin{aligned}
& \iota^{-}\left(y_{i}\right)=e^{\epsilon_{i}} \\
& \iota^{-}\left(\xi_{i}\right)=e^{-\epsilon_{i}}\left(\zeta_{i}-u \sum_{k<i}[k, i]\right) .
\end{aligned}
$$

Moreover, $\iota^{-}$is injective.

(2) The $\iota^{-}$extends to an algebra isomorphism:

$$
\mathbb{C}\left[\underline{y}^{ \pm}\right] \otimes_{\mathbb{C}[\underline{y}]} \ddot{\mathfrak{H}}^{-} \stackrel{\cong}{\longrightarrow} \ddot{\mathfrak{H}}_{\text {tr }}^{-}
$$


The inverse map $j^{-}$is an extension of the identity map on $\mathbb{C} S_{n}^{-}$such that

$$
\begin{aligned}
j^{-}\left(e^{\epsilon_{i}}\right) & =y_{i}, \\
j^{-}\left(\zeta_{i}\right) & =y_{i} \xi_{i}+u \sum_{k<i}[k, i] .
\end{aligned}
$$

Proof. Follows from Theorem 4.2 and Theorem 5.2; or one argues directly as for Theorem 5.2.

Theorem 5.6 and the PBW Theorem 3.2 for $\ddot{\mathfrak{H}}^{-}$immediately imply the following.

Corollary 5.7. The PBW property holds for $\ddot{\mathfrak{H}}_{t r}^{-}$. That is, the multiplication of the subalgebras gives rise to an isomorphism of vector spaces:

$$
\mathbb{C}[P] \otimes \mathbb{C} S_{n}^{-} \otimes \mathbb{C}\left[\zeta_{1}, \ldots, \zeta_{n}\right] \stackrel{\cong}{\longrightarrow} \ddot{\mathfrak{H}}_{t r}^{-} .
$$

5.3. An algebra isomorphism. The following theorem can be established by a direct computation.

Theorem 5.8. There exists a superalgebra isomorphism:

$$
\begin{aligned}
& \Phi^{t r}: \ddot{\mathfrak{H}}_{t r}^{\mathfrak{c}} \stackrel{\cong}{\longrightarrow} \mathcal{C}_{n} \bigotimes \ddot{\mathfrak{H}}_{t r}^{-} \\
& e^{\epsilon_{i}} \mapsto e^{\epsilon_{i}}, c_{i} \mapsto c_{i}, \epsilon_{i}^{\vee} \mapsto \sqrt{-2} c_{i} \zeta_{i}, s_{i} \mapsto \frac{1}{\sqrt{-2}}\left(c_{i}-c_{i+1}\right) t_{i} .
\end{aligned}
$$

The inverse map is given by

$$
e^{\epsilon_{i}} \mapsto e^{\epsilon_{i}}, c_{i} \mapsto c_{i}, \zeta_{i} \mapsto \frac{1}{\sqrt{-2}} c_{i} \epsilon_{i}^{\vee}, t_{i} \mapsto \frac{1}{\sqrt{-2}}\left(c_{i+1}-c_{i}\right) s_{i}
$$

Notes added. This first paper on spin Hecke algebras was posted in arXiv: math.RT/0608074, 2006. The results of this paper have been generalized and extended in different directions since then in a series of papers $[17,18,19,20,21]$.

\section{Acknowledgment}

This research is partially supported by NSF and NSA grants. I gratefully acknowledge the sesquicentennial Associateship from University of Virginia which allows me to spend the semester of Spring 2006 at MSRI, Berkeley. I thank MSRI for its stimulating atmosphere and support, where this work was carried out. I thank Ta Khongsap for his careful reading and corrections.

\section{References}

[1] J. Brundan and A. Kleshchev, Hecke-Clifford superalgebras, crystals of type $A_{2 l}^{(2)}$ and modular branching rules for $\widehat{S}_{n}$, Represent. Theory 5 (2001), 317-403 (electronic).

[2] I. Cherednik, Double affine Hecke algebras, London Mathematical Society Lecture Note Series 319, Cambridge University Press, 2005.

[3] V. Drinfeld, Degenerate affine Hecke algebras and Yangians, Funct. Anal. Appl. 20 (1986), $58-60$.

[4] C. Dunkl, Differential-difference operators associated to reflection groups, Trans. Amer. Math. Soc. 311 (1989), 167-183.

[5] P. Etingof and V. Ginzburg, Symplectic reflection algebras, Calogero-Moser space, and deformed Harish-Chandra homomorphism, Invent. Math. 147 (2002), 243-348. 
[6] I. Gordon, Baby Verma modules for rational Cherednik algebras, Bull. London Math. Soc. 35 (2003), 321-336.

[7] A. Kleshchev, Linear and projective representations of symmetric groups, Cambridge Tracts in Mathematics 163, Cambridge University Press, 2005.

[8] G. Lusztig, Affine Hecke algebras and their graded version, J. Amer. Math. Soc. 2 (1989), $599-635$.

[9] M. Nazarov, Young's symmetrizers for projective representations of the symmetric group, Adv. Math. 127 (1997), 190-257.

[10] A. Ram and A. Shepler, Classification of graded Hecke algebras for complex reflection groups, Comment. Math. Helv. 78 (2003), 308-334.

[11] R. Rouquier, Representations of rational Cherednik algebras, In: Infinite-dimensional aspects of representation theory and applications (Charlottesville 2004), Contemp. Math. 392 (2005), 103-131.

[12] K. Saito, Extended affine root system I. Coxeter transformations, Publ. Res. Inst. Math. Sci. 21 (1985), 75-179.

[13] I. Schur, Über die Darstellung der symmetrischen und der alternierenden Gruppe durch gebrochene lineare Substitutionen, J. reine angew. Math. 139 (1911), 155-250.

[14] A. Sergeev, The Howe duality and the projective representations of symmetric groups, Represent. Theory 3 (1999), 416-434.

[15] T. Suzuki, Rational and trigonometric degeneration of the double affine Hecke algebra of type A, Int. Math. Res. Not. 37 (2005), 2249-2262.

[16] M. Yamaguchi, A duality of the twisted group algebra of the symmetric group and a Lie superalgebra, J. Algebra 222 (1999), 301-327.

[17] T. Khongsap, Hecke-Clifford algebras and spin Hecke algebras III: The trignometric type, J. Algebra 322 (2009), 2731-2750.

[18] T. Khongsap and W. Wang, Hecke-Clifford algebras and spin Hecke algebras I: the classical affine type, Transformation Groups 13 (2008), 389-412.

[19] T. Khongsap and W. Wang, Hecke-Clifford algebras and spin Hecke algebras II: the rational double affine type, Pacific J. Math. 238 (2008), 73-103.

[20] T. Khongsap and W. Wang, Hecke-Clifford algebras and spin Hecke algebras IV: odd double affine type, Special Issue on Dunkl Operators and Related Topics, SIGMA 5 (2009), 012, 27 pages.

[21] W. Wang, Spin Hecke algebras of finite and affine types, Adv. in Math. 212 (2007), 723-748.

Department of Mathematics, University of Virginia, Charlottesville, VA 22904

E-mail address: ww9c@virginia.edu 